\title{
Aprendizado sobre envelhecimento humano na ótica de discentes de Enfermagem
}

\author{
Learning about human aging from the view of Nursing students \\ Aprender sobre el envejecimiento humano desde la perspectiva de estudiantes de Enfermería
}

\author{
Maxsuel Mendonça dos Santos \\ ORCID: https://orcid.org/0000-0002-0810-2525 \\ Universidade Federal do Rio Grande do Norte, Brasil \\ E-mail: maxsuelmendonca2016@gmail.com \\ Shirleíze Mariane Pereira Santos e Gomes \\ ORCID: https://orcid.org/0000-0002-2652-2515 \\ Universidade Federal do Rio Grande do Norte, Brasil \\ E-mail: shirleize_smps@hotmail.com \\ Flávia Rayonara Santana da Silva \\ ORCID: https://orcid.org/0000-0001-7004-1199 \\ Universidade Federal do Rio Grande do Norte, Brasil \\ E-mail: rayonara10@hotmail.com \\ Clara Caroline dos Santos Silva \\ ORCID: https://orcid.org/0000-0002-2607-947X \\ Universidade Federal do Rio Grande do Norte, Brasil \\ E-mail: claracaroline@ live.com \\ Jayara Mikarla de lira \\ ORCID: https://orcid.org/0000-0002-1707-0983 \\ Universidade Federal do Rio Grande do Norte, Brasil \\ E-mail: jayara.lira.104@ufrn.edu.br \\ Jocellem Alves de Medeiros \\ ORCID: https://orcid.org/0000-0002-6372-8424 \\ Universidade Federal do Rio Grande do Norte, Brasil \\ E-mail: jocellemmedeiros@gmail.com \\ José Jailson de Almeida Júnior \\ ORCID: https://orcid.org/0000-0001-7448-0703 \\ Universidade Federal do Rio Grande do Norte, Brasil \\ E-mail: jailson.junior@ufrn.br
}

\begin{abstract}
Resumo
Objetivo: Analisar as perspectivas de discentes no ensino e aprendizado do processo de envelhecimento humano com olhar a ação educadora. Metodologia: Trata-se de uma pesquisa de abordagem qualitativa do tipo exploratóriodescritivo. Sendo constituída por três fases: identificação e seleção de discentes aptos a participar do estudo, entrevistas individuais com roteiro de questões e gravação de respostas e transcrição das falas e respostas na íntegra para agrupamento de pensamentos. Resultados: Foi observado o desenvolvimento do processo ensino e aprendizado em vários aspectos inerentes a esse fenômeno com base nos olhares e subjetividade dos discentes, categorizando classes com âmbitos institucionais, docente, discente e subjetivos ao aprendizado e trabalho profissional no processo de envelhecimento humano. Conclusão: No contexto da pesquisa tornou-se evidente as potencialidades e dificuldades presentes nos aspectos educacionais sobre o processo de envelhecimento a partir da perspectiva discente, proporcionando oportunidade para se compreender como se constitui esse processo de formação e atuação em enfermagem.
\end{abstract}

Palavras-chave: Educação em enfermagem; Saúde do idoso; Aprendizagem; Educação superior.

\begin{abstract}
Objective: To analyze the perspectives of students in teaching and learning about the human aging process with a view to educational action. Methodology: This is an exploratory-descriptive qualitative approach research. Consisting of three phases: identification and selection of students able to participate in the study, individual interviews with script of questions and recording of answers and transcription of the speeches and answers in full for grouping thoughts. Results: The development of the teaching and learning process was observed in various aspects inherent to this phenomenon based on the views and subjectivity of students, categorizing classes with institutional, teacher, student and subjective scopes to learning and professional work in the human aging process. Conclusion: In the context of the research, the potential and difficulties present in the educational aspects of the aging process from the student perspective became evident, providing an opportunity to understand how this process of training and performance in nursing is constituted.
\end{abstract}

Keywords: Education nursing; Health of the elderly; Learning; Education higher. 


\begin{abstract}
Resumen
Objetivo: Analizar las perspectivas de los estudiantes en la enseñanza y el aprendizaje del proceso de envejecimiento humano con miras a la acción educativa. Metodología: Se trata de una investigación de enfoque cualitativo exploratorio-descriptivo. Consta de tres fases: identificación y selección de alumnos capaces de participar en el estudio, entrevistas individuales con guión de preguntas y grabación de respuestas y transcripción de los discursos y respuestas en su totalidad para agrupación de pensamientos. Resultados: Se observó el desarrollo del proceso de enseñanza y aprendizaje en diversos aspectos inherentes a este fenómeno a partir de las visiones y subjetividad de los estudiantes, categorizando las clases con ámbitos institucional, docente, estudiantil y subjetivo al aprendizaje y trabajo profesional en el proceso de envejecimiento humano. Conclusión: En el contexto de la investigación, se evidenciaron las potencialidades y dificultades presentes en los aspectos educativos del proceso de envejecimiento desde la perspectiva del estudiante, brindando la oportunidad de comprender cómo se constituye este proceso de formación y desempeño en enfermería.
\end{abstract}

Palabras clave: Educación en enfermería; Salud de los ancianos; Aprendiendo; Educación universitária.

\title{
1. Introdução
}

A perspectiva de envelhecimento se faz cada vez mais presente no contexto mundial, há estimativa que no ano de 2050 ocorra uma abrangência próxima de dois bilhões de pessoas com sessenta anos ou mais no mundo, boa parte integrando países que se encontram em desenvolvimento, compondo a necessidade do planejamento em ações e políticas para atender as demandas emergentes. Configura-se tal associação no aumento de expectativa de vida devido a alterações em indicadores como saúde, queda de fecundidade e mortalidade proporcionando novos olhares aos desafios que se erguem. (Brasil, 2006; Veras \& Oliveira, 2018)

O envelhecimento pode ser compreendido entre o processo de senescência e senilidade, sendo o primeiro um processo natural do corpo com tendência a não ocasionar problemas ao corpo e o segundo sendo entendido como um processo patológico em decorrência de algum tipo de doença, acidente e/ou estresse emocional, passível da necessidade de assistência (Brasil, 2006).

Nesse contexto, a formação profissional nas instituições de ensino superior possuem como recomendações e necessidade a implementação de ensino aos princípios inerentes do cuidar ao idoso e sua saúde, a exemplo, independência e autonomia, divergindo de realidades onde estas não se incluem nas grades curriculares da área da saúde mesmo demonstrando sua relevância para a formação (Carvalho, 2015).

As ações educativas de promoção a saúde do idoso também se demonstram incipientes em pesquisas científicas sendo observado a necessidade de inovações para esta área a partir de um processo criativo que proporcione autonomia e participação ativa para todos, atentando a soluções que supram as necessidades dos idosos (Mallman, et al., 2015).

A educação popular e a promoção da saúde na perspectiva da oferta de um envelhecimento ativo e saudável segundo Souza, Silva e Barros (2021) demonstram déficit e limitações quanto a integração a ações junto à comunidade havendo segregação etária e falta de entendimento quanto considerar o envelhecimento como processo contínuo e pertinente a todos.

O aprendizado e o desenvolvimento de ações que promovam à saúde do idoso devem compreender e contemplar o processo de envelhecimento, associado a fatores inerentes ao indivíduo e sua subjetividade, trazendo inovações que possuam como foco central o idoso no seu entendimento holístico para que possa se alcançar as metas necessárias a formação (Mallman, et al.,2015).

A percepção de discentes de enfermagem em sua formação no aprendizado do cuidado ao paciente idoso e o processo de envelhecimento, podem contribuir para a compreensão nos métodos empregados nos papéis de ensino e aprendizagem, evidenciando metodologias, necessidades e soluções para a construção deste conhecimento nas áreas de formação em saúde, constituindo quebras para paradigmas já instituídos. 
A Partir do exposto, objetiva-se compreender as perspectivas discentes acerca do fenômeno ensino e aprendizagem quanto o processo de envelhecimento humano no entendimento as prerrogativas concernentes ao desenvolvimento do processo educador.

\section{Metodologia}

Trata-se de uma pesquisa de abordagem qualitativa do tipo exploratório-descritivo. A partir da realização de entrevistas individuais com o objetivo de compreender os aspectos de forma integral da subjetividade na construção dos significados e valores dos indivíduos (Minayo, 2017).

A pesquisa se desenvolveu em uma universidade do interior do estado do Rio Grande do Norte em agosto de 2016, sendo constituída por três fases, a primeira fase composta por identificação e seleção de discentes aptos a participar do estudo, a segunda fase com foco na realização de entrevistas individuais com implementação de roteiro de questões e gravação de respostas e a terceira fase na transcrição das falas e respostas na íntegra dos discentes participantes (Bardin, 2016), para agrupamento de pensamentos com a ferramenta IRaMuTeQ versão 0.7 alpha 2 na interpretação e diálogo de dados (Souza, et al., 2018).

Foram considerados a inserção de setenta e cinco discentes do qual a amostragem se compôs com vinte e um discentes a partir da avaliação e delimitações dos critérios de inclusão, sendo estar matriculado e ativo no curso de graduação de Enfermagem, ter cursado o componente curricular de Atenção Básica e Saúde da Família, estar apto a responder perguntas de caráter subjetivo, concordar em participar da pesquisa e assinar o Termo de Consentimento Livre e Esclarecido (TCLE) e o termo de autorização para gravação de voz.

A coleta sucedeu a partir de planejamento, elaboração e implementação de roteiro semiestruturado para as entrevistas individuais com questionamentos relevantes que incentivassem ao pensamento crítico acerca dos processos educacionais e de formação para a construção do conhecimento sobre o processo de envelhecimento, o estímulo as práticas desenvolvidas nos setores institucionais de saúde e os sentimentos pertencentes as vivências.

A pesquisa foi fundamentada segundo os princípios éticos da Resolução no 466/2012 do Conselho Nacional de Saúde sobre pesquisa envolvendo seres humanos, cumprindo todas as exigências e regulamentações específicas contidas nesta resolução. O projeto foi aprovado pelo CEP/FACISA através do parecer $\mathrm{n}^{\circ} 1.439 .099$. Para preservar a integridade dos participantes ao longo dos resultados e discussão as falas serão identificadas pela palavra "DISCENTE" seguido de um número de ordem.

\section{Resultados e Discussão}

As práticas de ensino ativas e inovadoras tendem a perpassar pela reflexão coletiva, o diálogo, a compreensão da realidade e das novas perspectivas se constituindo como base ao desenvolvimento de novos caminhos ao aprendizado (Barros, et al., 2017). A problematização nesse contexto, integra-se na promoção do agir subjetivo na autonomia, reflexão e criticidade, assim, metodologias ativas surgem na formação profissional de discentes favorecendo a dinamicidade na resolução dos mais diversos cenários em saúde (Leal, et al., 2018).

A compreensão, portanto, se faz necessário por meio da perspectiva dos discentes e como se configura o entendimento para estes através das teorias e experiências, que devem ser entendidas como significado de reavivar e tornar vivo o fenômeno como objeto de recurso adequado a elucidação das perspectivas (Bastos, 2017).

O processamento das falas e resposta obteve como retorno a formulação de três eixos temáticos principais, sendo constituídos a partir de seis classes significativamente relevantes das entrevistas; o eixo de número um compreendia aspectos associados ao aprendizado e a instituição de ensino abrangendo a área de educação, o segundo eixo se demonstra envolto nos 
aspectos do desenvolvimento de emoções e sentimentos compondo a parte subjetiva associados ao processo de envelhecimento e o eixo de número três abarcando as instituições de serviços em saúde no desenvolvimento de ações e intervenções formando a área de assistência à saúde. A partir da necessidade de haver uma assistência qualificada ao idoso e com o entendimento que está concretiza-se no encontro da formação profissional (Brasil, 2006; Souza, et al., 2021), o eixo de número um compreendendo a educação se torna evidente como foco para a discussão da pesquisa.

Nos contextos de ensino em enfermagem, se tem o conhecimento que o ensino sobre o processo de envelhecimento se encontra de forma escassa, se resumindo a iniciativas isoladas de professores que demonstram interesse no ensino de tais conhecimentos observando relevância para o diálogo em aulas e a implementação em campos de prática voltados ao público idoso, trazendo para a formação também aspectos sociais e constitucionais associados a terceira idade, constituindo conhecimento para o desenvolvimento qualificado em saúde profissional para tal população (Freitas \& Mendes, 2003; Carvalho, 2015). Observa-se tais configurações nas interjeições dos discentes.

"Na universidade a gente tem algumas matérias que falam do envelhecimento só que não é aquela coisa, é para lidar no dia a dia, é uma coisa mais por cima [...]" (DISCENTE_09)

"A universidade não costuma nos preparar muito bem para essa parcela da população, a gente geralmente é mais preparado para lidar com a faixa etária de jovens e adulto, mas quando parte para crianças e idosos a gente sente uma necessidade muito grande de melhorar pra o estudante saber lidar com esta parcela" (DISCENTE_17)

A perspectiva de construção do conhecimento a partir da interação com o outro, sendo orientado pelo docente possuidor do papel de contribuir na transformação da informação em conhecimento, lançando mão de ferramentas para tal tarefa enxergada de forma complexa pelos alunos, se apresenta com dificuldade no processo de assimilação e autonomia na organização e construção do próprio conhecimento. Havendo a necessidade de entender que tais desafios provêm de um processo educacional imposto de forma vertical, ou seja, a educação bancária onde há o depósito de informações, porém não ocorre o desenvolvimento crítico e reflexivo do aprendizado sendo a sala de aula vista como local propício para tal construção na perspectiva discente. (Freire, 2011b; Brighente \& Mesquida, 2016).

"Eita!, Eu não sei de nada disso aqui, eu acho que foi passado por cima eu não lembro mas de nada, prepara mas assim precisa trabalhar bem mais em sala de aula" (DISCENTE_07).

"Não tem aquele preparo realmente com o idoso, a gente chega num sabe como conversar, não sabe como chegar porque tem pouca prática e o conteúdo que é dado em sala de aula é muito jogado não prepara a gente[...]" (DISCENTE_09)

Assim é evidente que o papel do professor é fundamental e perpassa por vários aspectos como a criatividade e elaboração de estratégias e ambientes de aprendizagem no tocante a contribuir na construção da informação em conhecimento, trazendo novos olhares a escola para que não se transforme em espaço de troca para informações sem finalidades, mas para construção do conhecimento. O reconhecimento na atuação do professor é percebido por seu educando mesmo que este não venha a ser expresso. (Brighente \& Mesquida, 2016; Martins, et al., 2018).

"O campo pra aula prática ele é bem reduzido, num da para gente aprender o que tem que ser aprendido, eu acho que a professora desse campo que trabalhou com a gente ela fez muito mais do que a realidade em si acontece, né" (DISCENTE_08).

A autonomia com olhar para o conhecimento se demonstra relevante para que o discente possa buscar pelo próprio conhecimento, com perspectivas ao enfermeiro devido a necessidade de se manter em constante atualização quanto as práticas 
assistenciais e o desenvolvimento do raciocínio crítico para tomada de decisão, carregando a responsabilidade por tal ato com base em evidências para atuação tanto assistenciais como administrativas; sendo estímulo o diálogo como transformadora da realidade a partir do contexto do indivíduo por meio da problematização de ideias (Freire, 2011a; Castro \& Malavasim, 2017), a compreensão de inacabamento por parte do aluno se faz presente a partir de seus pensamentos.

"Então, eu acho que vai muito da percepção do aluno também correr atrás e procurar aprender um pouco mais sobre esse assunto" (DISCENTE_10).

"Quando tem que trabalhar com a pessoa idosa chega o momento e você tem que desenrolar, você leva sim grande conhecimento teórico, mas grande parte aprende lá na hora quando você tem que lidar com essa pessoa" (DISCENTE_13)

Portanto para que o aluno de enfermagem consiga elaborar tal autonomia à necessidade de entender que não se retêm todo o conhecimento e a compreensão de se estar inacabado havendo sempre algo a se aprender, possuindo os professores contribuição nos aspectos do diálogo problematizador e crítico com ênfase abordados em conteúdos e experiências práticas da realidade (Castro \& Malavasim, 2017).

$\mathrm{O}$ ambiente institucional voltado a oferta de ensino de qualidade remete como um dos fatores essenciais as relações desenvolvidas entre os envolvidos aluno-turma, aluno-docente e aluno-funcionário sendo condição importante na oferta de ensino qualificado (Dourado \& Oliveira, 2009; Pereira, 2017). Neste contexto a interjeição a seguir consegue demonstrar como se procede os aspectos relacionais como facilitador do aprendizado.

“[...] eu creio que a faculdade contribui com isso e ela disponibiliza professores capacitados que desde o início da minha faculdade puderam trabalhar com a gente essa temática, disponibilizando meios em que a gente pode se capacitar" (DISCENTE_19)

Nesse relato nota-se a percepção do discente com relação as contribuições que a faculdade consegue oferecer através da inserção de professores capacitados que através das relações aluno-docente constitui ferramentas capazes de ajudar no aprendizado da capacitação.

A partir dos relatos houve dicotomia associados ao ato de se estar preparado para a atuação profissional, possuindo sentimento de insegurança e falta de capacidade, assim como sentimentos de confiança e necessidade de atuação expressados nos pensamentos.

"Então eu acho assim que nós ainda não estamos preparados, pelo menos eu não estou preparado como aluno de enfermagem para lidar com essas situações especificas[...]” (DISCENTE_02).

"A sensação que eu como aluna tenho e que eu acredito que muitos alunos pensem, é que a gente não está preparado que a gente não sabe de tudo que a gente quando chegar na realidade a gente não vai conseguir fazer" (DISCENTE_08).

Percebe-se nessas interjeições a constância no sentimento de não estar preparado para se atuar na assistência ao idoso, nas características de conhecimento insuficiente sobre saúde do idoso e a incapacidade de atuar nas práticas assistências para essa população específica. No entanto houve pensamentos que evidenciassem o contrário formadores da dicotomia.

"Nos periodos finais do curso a gente é mais preparado pra lidar com a prática do que é, como é que eu posso dizer, vivenciar essa realidade preparar pra vivenciar essa realidade" (DISCENTE_07) 
No entanto, ressalta-se que o sentimento de estar preparado se elucida próximo a finalização da graduação, onde já ocorreu maiores experiências em campos de práticas e o acúmulo de vivências tende a gerar confiança nas atuações seguintes (Negreiros \& Lima, 2018).

A inserção de disciplinas que abordem tal processo favorece a eliminação de preconceitos e estereótipos associados a população idosa, capacitando profissionais competentes no exercício de sua profissão (Carvalho, 2015). O envelhecimento a partir da construção e representação social, demonstra efeitos negativos e que devem ser abordados em disciplinas nas instituições de ensino, nessa perspectiva as interjeições a seguir demonstram como se deu tal processo na construção social.

"[...] Todos os aspectos biológicos, é como é que vai acontecendo com o envelhecimento, ou seja, é a perda de alguns reflexos de alguns movimentos" (DISCENTE_08)

"Um dia ele (idoso) vai estar afetuoso e no outro dia vai estar raivoso essas mudanças de humor drásticas decorrentes também da situação do envelhecimento e da patologia dele, você tem que saber lidar, saber lidar com aquela situação" (DISCENTE_13)

O ageísmo estruturado com base na sistemática de estereótipos e preconceitos a partir da idade, impactam negativamente a qualidade de vida do idoso; a universidade demonstra-se como parte instrumental na criação e implementação de espaços que apresentem como objetivo o desenvolvimento e transformação social, categorizando a inclusão da população idosa nas relações intergeracionais elevando a qualidade de vida e reduzindo o ageísmo (Neto, et al., 2020).

O processo de envelhecimento e a oferta de assistência qualificada em saúde ao idoso, demandam de um ensino e aprendizado que desenvolvam o raciocínio crítico e problematizar, com o uso de criatividade e diálogo para compreensão desse processo, constituindo abertura para novas perspectivas e alterações de paradigmas estruturais, incentivando ao fim a possibilidade de uma assistência integral e equânime ao idoso.

\section{Conclusão}

No contexto da pesquisa tornou-se evidente as potencialidades e dificuldades presentes nos aspectos educacionais sobre o processo de envelhecimento a partir de uma perspectiva advinda do olhar discente, proporcionando oportunidade para se compreender como se constitui esse processo de formação e atuação em enfermagem.

Portanto, se configurou os processos institucionais na oferta de ensino de qualidade a partir das interações entre sujeitos e disponibilização de professores qualificados que venham propor a construção do conhecimento e orientação de informações para o diálogo crítico e problematizador. No entanto se ressalta a necessidade de formular disciplinas capazes de trabalhar em formação o entendimento do processo de envelhecimento abrindo espaço para preparo e capacitação profissional discente proporcionando confiança ao se trabalhar com a assistência ao idoso.

A autonomia altamente associada ao ensino, demonstrou aspecto fundamental na construção como aluno e enfermeiro, necessitando desenvolver o estímulo a busca pelo conhecimento e que o indivíduo também é parte fundamental no processo de aprendizado, para o aluno com funcionalidade de construir-se como profissional atuante e conhecedor das disciplinas inerentes a sua área e para o profissional enfermeiro como habilitadora para tomada de decisão com embasamento científico e atualizado proporcionando melhor assistência ao paciente.

Sentimentos de se encontrar apto e preparado para a atuação profissional se fazem presente quando existe o encontro com a realidade em si, havendo dúvidas acerca do aprendizado vivenciado em sala de aula, sendo eficazes e suficiente para atuar e o oposto ocorre com base nas mesmas circunstâncias num entendimento de confiança e segurança na atuação.

A implementação de forma adequada no ensino ao processo de envelhecimento possui papel divisor de paradigmas, associados ao olhar preconceituoso e estereotipado construído socialmente sobre o envelhecer e se tornar idoso, associado em 
muitos casos ao surgimento de patologias e comorbidades, instituindo então um olhar sobre o envelhecimento ativo e saudável que não necessariamente vem associado a quadros patológicos.

O processo de envelhecimento constitui conhecimentos complexos e específicos pertencentes ao humano, faz-se necessário o incentivo a novas pesquisas que consigam trazer olhares ao desenvolvimento de disciplinas voltadas ao envelhecer de forma mais presente nas instituições de ensino superior em saúde, trabalhando na perspectiva de ensino problematizador e de construção mútua de conhecimento, abrindo novos horizontes a formação profissional e a assistência à saúde do idoso.

\section{Referências}

Bardin, L. (2016). Análise de Conteúdo. Edições 70.

Barros, K. B. N. T., dos Santos, S. L. F., \& Lima, G. P. (2017). Perspectivas da Formação no Ensino Superior Transformada Através de Metodologias Ativas: Uma Revisão Narrativa da Literatura. Revista Conhecimento Online, 1, 65-76. https://periodicos.feevale.br/seer/index.php /revistaconhecimentoonline/article/view/472.

Bastos, C. C. B. C. (2017). Pesquisa qualitativa de base fenomenológica e a análise da estrutura do fenômeno situado: algumas contribuições. Revista Pesquisa Qualitativa, 5(9), 442-451. https://editora.sepq.org.br/rpq/article/view/156.

Brasil (2006). Envelhecimento e saúde da pessoa idosa. Brasília: Ministério da Saúde. https://bvsms.saude.gov.br/bvs/publicaco es/evelhecimento_saude_pessoa_idosa.pdf.

Brighente, M. F., \& Mesquida, P. (2016). Paulo Freire: da denúncia da educação bancária ao anúncio de uma pedagogia libertadora. Pro-Posições, 27(1), 155177. 10.1590/0103-7307201607909.

Carvalho, C. R. A. D. (2015). A saúde do idoso no ensino superior de universidades públicas do Rio de Janeiro: o caso dos cursos de Educação Física, Enfermagem e Nutrição. Tese de doutorado - Escola Nacional de Saúde Pública Sérgio Arouca, Rio de Janeiro, Brasil. https://www.arca.fiocruz.br/handle/icict/12983

de Castro, S. P., \& Malavasim, A. (2017). a relação da pedagogia da autonomia de paulo freire com a prática docente no contexto educacional. $e$ Mosaicos, 6(13), 105-111. 10.12957/e-mosaicos.2017.30808.

Dourado, L. F., \& Oliveira, J. F. D. (2009). A qualidade da educação: perspectivas e desafios. Cadernos Cedes, 29(78), 201-215. 10.1590/S010132622009000200004

Freire, P. (2011a). Extensão ou comunicação? (15a ed.). Paz e Terra.

Freire, P (2011b). Pedagogia da autonomia: saberes necessários à prática educativa. Paz e Terra.

Freitas, M. C. D., \& Mendes, M. M. R. (2003). O ensino sobre o processo de envelhecimento e velhice nos cursos de graduação em enfermagem. Revista Brasileira de Enfermagem, 56(5), 502-507. 10.1590/S0034-71672003000500007.

Leal, L. B., Pereira, K. L. A., Negreiros, A. L. B., Pequeno, A. M. C., Lima, G. P., Negreiros, F. D. D. S., \& Leitinho, M. C. (2018). Método ativo problematizador como estratégia para formação em saúde. Rev. enferm. UFPE on line, 1139-1143. https://pesquisa.bvsalud.org/portal/resource/pt/biblio970817

Mallmann, D. G., Galindo Neto, N. M., Sousa, J. D. C., \& Vasconcelos, E. M. R. D. (2015). Educação em saúde como principal alternativa para promover a saúde do idoso. Ciência \& Saúde Coletiva, 20, 1763-1772. 10.1590/1413-81232015206.02382014.

Martins, E. D., Moura, A. A., \& de Araújo Bernardo, A. (2018). O processo de construção do conhecimento e os desafios do ensino-aprendizagem. Revista on line de Política e Gestão Educacional, 410-423. 10.22633/rpge.v22.n.1.2018.10731.

Minayo, M. C. D. S. (2017). Cientificidade, generalização e divulgação de estudos qualitativos. Ciência \& Saúde Coletiva,22, 16-17. 10.1590/141381232017221.30302016

Negreiros, R. V., \& de Lima, V. C. B. (2018). Importância do estágio supervisionado para o acadêmico de enfermagem no hospital: compartilhando experiências vivenciadas com a equipe de trabalho. Revista da Universidade Vale do Rio Verde, 16(2). 10.5892/ruvrd.v16i2.4359.

Neto, M. J. R., Rezende, M. C. R. A., de Oliveira Limírio, J. P. J., Dalben, A. C., Rezende, M. I. R. A., Pescinini-Salzedas, L. M., \& Salzedas, L. M. P. (2020). Estereótipos sobre os idosos: o papel da Universidade na redução do ageismo. archives of health investigation, 9(1). 10.21270/archi.v9i1.5098.

Pereira, J. C. (2017). Afetividade: A importância da relação professor e aluno como fator motivacional no processo de ensino e aprendizagem. Monografia/TCC - Universidade Federal da Paraíba, João Pessoa, Paraíba, Brasil. http://www.ccen.ufpb.br/cccb/contents/monografias/monografias2017/jalcines-da-costa-pereira.pdf.

Souza, E. M. D., Silva, D. P. P., \& Barros, A. S. D. (2021). Educação popular, promoção da saúde e envelhecimento ativo: uma revisão bibliográfica integrativa. Ciência \& Saúde Coletiva, 26, 1355-1368. 10.1590/1413-81232021264.09642019. 
Research, Society and Development, v. 10, n. 13, e252101320984, 2021

(CC BY 4.0) | ISSN 2525-3409 | DOI: http://dx.doi.org/10.33448/rsd-v10i13.20984

Souza, M. A. R. D., Wall, M. L., Thuler, A. C. D. M. C., Lowen, I. M. V., \& Peres, A. M. (2018). O uso do software IRAMUTEQ na análise de dados em pesquisas qualitativas. Revista da Escola de Enfermagem da USP, 52. 10.1590/S1980-220X2017015003353.

Veras, R. P., \& Oliveira, M. (2018). Envelhecer no Brasil: a construção de um modelo de cuidado. Ciência \& saúde coletiva, 23, 1929-1936. 10.1590/141381232018236.04722018 . 